\title{
An Atypical Presentation of Chylous Ascites
}

${ }^{1}$ TR Tanusha, ${ }^{2}$ Mamatha B Patil

\begin{abstract}
Chylous ascites (CA) is the extravasation of milky chyle into the peritoneal cavity. Chylous ascites commonly affects adults in 50 to 60 years of age but can also occur in pediatric population. In adults, the most common causes are abdominal malignancies and cirrhosis which account for more than two-third of the cases in developed countries, whereas infectious diseases, such as tuberculosis and filariasis, are prevalent in developing countries. Other causes include congenital, inflammatory, postoperative, traumatic and miscellaneous disorders. We hereby report two atypical cases of chylous ascites and also briefly discuss the causes and treatment.
\end{abstract}

Keywords: Adenosine deaminase, Antitubercular treatment, Chylous ascites, Contrast-enhanced computed tomography.

How to cite this article: Tanusha TR, Patil MB. An Atypical Presentation of Chylous Ascites. J Med Sci 2015;1(1):17-20.

Source of support: Nil

Conflict of interest: None

\section{INTRODUCTION}

Chyle is grossly milky in appearance similar to the peripheral lymph. In vitro characteristics of chylous fluid are its separation into a creamy layer on standing, alkaline $\mathrm{pH}$, specific gravity between 1.010 and 1.054, total protein that varies from 1.4 to $6.4 \mathrm{gm} / \mathrm{dl}$, fat content of 0.4 to $4 \%$ total solids, $14 \%$ positive staining of fat globules with Sudan red stain and normal glucose, amylase and cholesterol. Chylous ascites (CA) contains high triglycerides usually higher than $200 \mathrm{mg} / \mathrm{dl}$. Some authors have identified an elevated plasma triglyceride ratio range between $2: 1$ and $8: 1$ as being indicative of $C A$. Chyliform ascites contains lecithin-globulin complex due to fatty degeneration of cells, and pseudochylous ascites fluid is milky in appearance due to the presence of pus. ${ }^{2}$

\section{CASE REPORTS}

\section{Case One}

A 40-year-old female presented with complaints of abdominal distension and easy fatiguability since

\footnotetext{
${ }^{1}$ Postgraduate Student, ${ }^{2}$ Professor and Unit Chief (IV)

1,2Department of General Medicine, RajaRajeswari Medical College \& Hospital, Bengaluru, Karnataka, India
}

Corresponding Author: Mamatha B Patil, Professor and Unit Chief (IV), Department of General Medicine, RajaRajeswari Medical College \& Hospital, Bengaluru, Karnataka, India Phone: 9845680586, e-mail: dr.mamatharamesh@yahoo.co.in
15 days. She had undergone appendicectomy 3 years back. There was no history of alcohol consumption or any other substance abuse. On examination, she looked emaciated, temporal wasting was noticed, body mass index (BMI) was $16 \mathrm{~kg} / \mathrm{m}^{2}$, vitals were stable and no lymphadenopathy. Abdomen was distended, fluid thrill was present. No organomegaly appreciated. Paracentesis was done, and milky white ascitic fluid was drained (Figs 1 and 2). On subjecting the fluid to investigations, it showed sugars of $165 \mathrm{mg} / \mathrm{dl}$, lactate dehydrogenase (LDH) $185 \mathrm{U} / \mathrm{L}$, triglycerides of $1188 \mathrm{mg} / \mathrm{dl}$, cholesterol $105 \mathrm{mg} / \mathrm{dl}$, adenosine deaminase (ADA) 6.6. Smear showed occasional lymphocytes and cholesterol crystals. Sediment smears showed lymphocytes in lipoidal and proteinaceous background. few epithelial cells and pus cells were also found. Acidfast bacillus (AFB) was negative and there was no growth on incubation for 48 hours.

Her complete blood count and renal parameters were within normal limits. Serum bilirubin was $0.2 \mathrm{mg} / \mathrm{dl}$, direct bilirubin was $0.1 \mathrm{mg} / \mathrm{dl}$, SGOT was 22IU, SGPT was 15IU, alkaline phosphate was 102IU, total protein was $5.6 \mathrm{mg} / \mathrm{dl}$, albumin was $2.9 \mathrm{mg} / \mathrm{dl}$ and globulin was $2.7 \mathrm{mg} / \mathrm{dl}$.

Human immunodeficiency virus (HIV) and HBSAg tests were negative. Upper GI endoscopy was normal. Transthoracic echo showed an EF of $64 \%$. Contrastenhanced computed tomography (CECT) of abdomen showed cross fused ectopic kidney, massive ascites with mesenteric lymphadenopathy. Tuberculin skin test was negative.

Around 4 liters of ascitic fluid was drained on alternative days with oral protein supplementation and discharged from the hospital. Patient was started on antitubercular treatment (AKT) along with steroids.

\section{Case Two}

A 55-year-old lady presented with complaints of pain in abdomen and abdominal distension and bilateral pitting pedal edema, weight loss of more than $10 \mathrm{~kg}$ since 2 months. She was diagnosed as a case of leprosy 20 years back and had completed treatment for the same. She had no known comorbidities. On clinical examination, she appeared pale. Bilateral pitting pedal edema was noticed. Loss of distal phalanges of all fingers of both hands with contracture at interphalangeal joints, BMI $24 \mathrm{~kg} / \mathrm{m}^{2}$. Peripheral blood smear showed microcytic hypochromic 


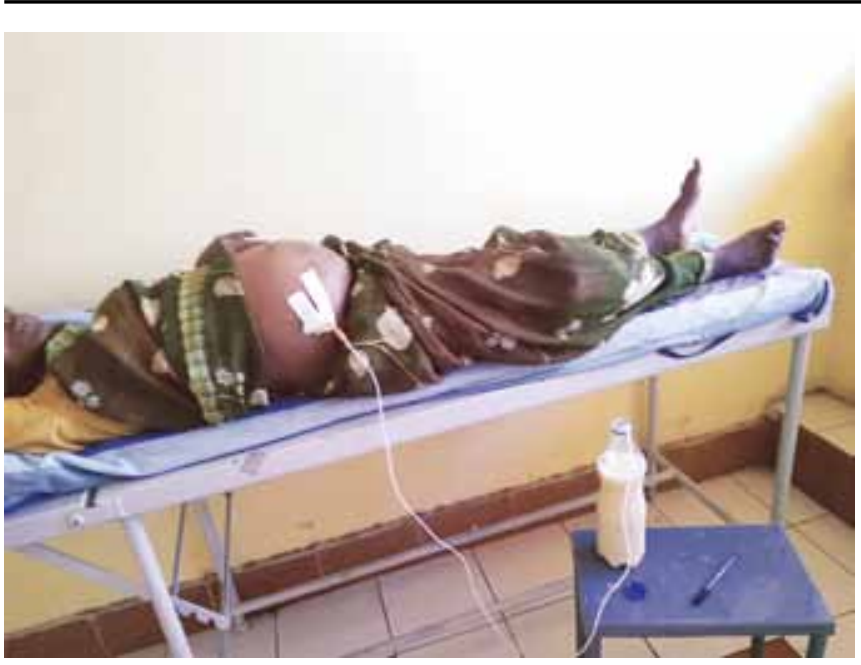

Fig. 1: Paracentesis

anemia with $\mathrm{Hb}$ of $9 \mathrm{gm} \%$. Liver and renal function tests were within normal limits. Ultrasonography (USG) of abdomen showed enlarged pre, para-aortic group of lymph nodes largest measuring $2 \mathrm{~cm}$. Enlarged inguinal group of lymph nodes and minimal ascites. Ultrasonography-guided ascitic tapping was done. Creamy white ascitic fluid was drained and sent for investigations. It showed proteins of $46 \mathrm{mg}$, sugar $108 \mathrm{mg}$, LDH 329, cholesterol 65 and triglycerides 379 . Cell count 80 out of which $90 \%$ were lymphocytes, $5 \%$ neutrophils, $5 \%$ mesothelial cells (lymphocytes with scattered neutrophils and mesothelial cells in a proteinaceous background).

Arterial and venous Doppler of lower limb only showed subcutaneous edema with no venous pathology. Split skin smear was negative for Mycobacterium leprae. Both patients were identified as tuberculosis etiology and started on AKT4 with steroids. Both patients came for follow-up after 4 weeks of AKT, clinical improvement noticed (increase in appetite, weight and decrease in abdominal girth).

\section{DISCUSSION}

Chylous ascites is defined as the extravasation of milky or creamy appearing peritoneal fluid rich in triglycerides, caused by the presence of thoracic or intestinal lymph in the abdominal cavity. ${ }^{1}$ It is a manifestation of underlying pathology and is not a disease by itself.

Morton, in 1691, performed paracentesis in an 18-month-old boy with disseminated tuberculosis and noted the presence of CA. ${ }^{2}$ Trauma was recognized as the most common cause in the 17th century. Later on, multiple causes have been described; these are abdominal surgery, blunt abdominal trauma, malignant neoplasm, spontaneous bacterial peritonitis, cirrhosis, pelvic irradiation, peritoneal dialysis, abdominal tuberculosis, carcinoid syndrome, and congenital defects of lacteal formation. In tropical countries, tuberculosis and

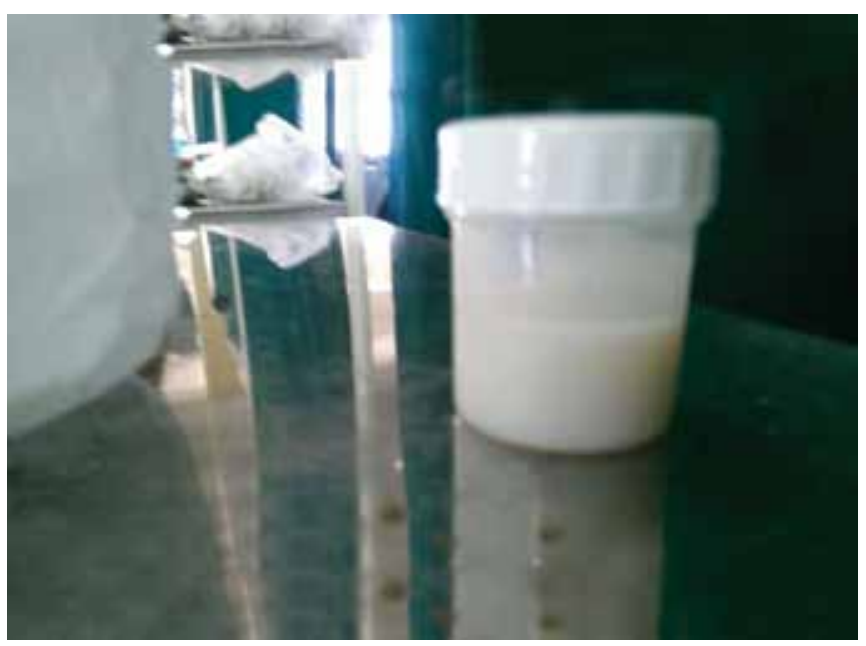

Fig. 2: Milky white chylous fluid

filariasis are the leading causes. Congenital abnormalities of the lymphatic system and trauma should be considered as the most important etiological factor in children..$^{3-5}$

A study was conducted on 35 patients to know the etiology and prognosis of CA. More than $90 \%$ cases were due to malignancy that included mainly lymphomas and abdominal carcinoma, cirrhosis and retroperitoneal surgery. These cases of malignant CA chiefly presented with extensive tumor dissemination with 1 year mortality rate of $80 \%$. Cases of CA with cirrhosis were having refractoriness to treatment in 13 out of 15 cases with mortality rate $69 \%$. Mycobacteria species is the most common cause of CA in HIV-infected patients. ${ }^{5-7}$

The possible mechanisms of CA are lymph node fibrosis or infiltration by malignancy obstructing the flow of chyle, leaking of chyle through a fistula into the peritoneal cavity due to congenital lymphangiectasia, and acquired thoracic duct obstruction with a lymphoperitoneal fistula causing chyle leakage from subserosal lymphatics. Paradoxical immune reconstitution inflammatory syndrome (IRIS) has also been documented in the development of CA. ${ }^{8}$

The clinical presentation of $\mathrm{CA}$ is characterized by progressive and painless abdominal distension, weight gain, signs of malnutrition along with symptoms, and signs of underlying conditions. Patient may also have chylothorax due to reflux of chyle across the diaphragm into the pleural cavities. Rarely, patients may have acute onset of CA with rapid weight gain and dyspnea, especially in a patient who has undergone abdominal and thoracic surgery. ${ }^{9,10}$

Patients of CA should be advised for complete blood count, renal function tests, liver function tests, trigly-cerides, cholesterol, amylase, lipase, and lactate dehydrogenase (LDH). They also should be advised to undergo ascitic fluid examination for biochemical [(triglycerides level, cholesterol level, ascitic albumin gradient (SAAG) 
ratio), cytological (cell count, cell types, malignant/atypical cells)] and microbiological examination like gram stain, culture sensitivity and AFB bacilli echocardiography. ${ }^{11,12}$ Computed tomography of the abdomen is useful (Fig. 3) in identifying pathological intra-abdominal lymph nodes and masses. Lymphangiography is the gold standard to define causes of lymphatic obstruction, abnormal retroperitoneal nodes, leakage from dilated lymphatics, and fistulization but has several complications related to the volume and type of contrast used. Lymphoscintigraphy is a less-invasive alternative to lymphangiography and can confirm an active lymphatic leak into the peritoneal cavity. These tests may be useful in selecting patients for surgery. Other investigations that are useful in CA are lymph node biopsy, laparoscopy or laparotomy, bone marrow examination, and intravenous pyelography. Chyle is grossly milky in appearance similar to the peripheral lymph. In vitro characteristics of chylous fluid are its separation into a creamy layer on standing alkaline $\mathrm{pH}$ specific gravity between 1.010 and 1.054 total protein that varies from 1.4 to $6.4 \mathrm{gm} / \mathrm{dl}$ fat content of 0.4 to $4 \%$ total solids $14 \%$, positive staining of fat globules with Sudan red stain and normal glucose amylase and cholesterol. Chylous ascites contains high triglycerides usually higher than $110 \mathrm{mg} / \mathrm{dl}$. Some authors have identified an elevated ascites, plasma triglyceride ratio between 2:1 and 8:1 as being indicative of CA. Chylous ascites is differentiated from pseudochyle and chyliform ascites by the presence of high triglyceride content that is low in pseudochyle and chyliform ascites.

Chylous ascites can be managed by treating the underlying cause. In most conditions, management of underlying condition, especially infective, inflammatory or hemodynamic causes, results in complete resolution of CA. ${ }^{5}$

Nonpharmacological treatments include dietary modification with salt and water restriction, elevation of legs and stocking. Dietary modifications include highprotein diet and use of a low-fat diet with medium-chain triglyceride supplementation that reduces the production and flow of chyle. This is because medium-chain triglycerides are absorbed directly into the intestinal cells and transported as fatty acids and glycerol directly to liver via the portal vein, whereas long-chain triglycerides need conversion to monoglycerides and free fatty acids that are transported as chylomicrons to the intestinal lymph ducts. Orlistat can be tried when patient have difficulty in complying with a low-fat diet. If CA persists despite dietary

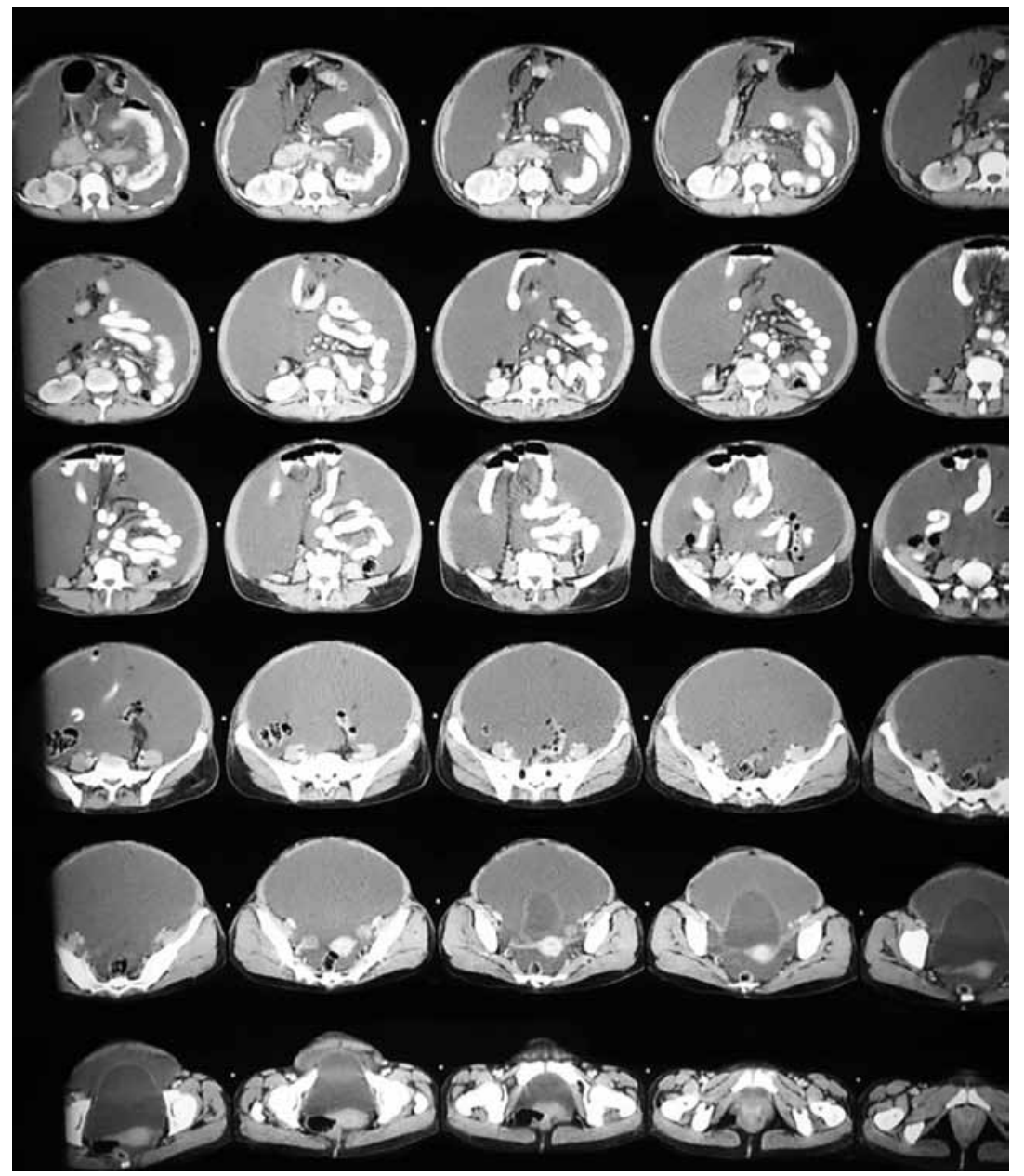

Fig. 3: Contrast-enhanced computed tomography of abdomen showing massive ascites with mesenteric lymphadenopathy 
management, the next step may involve bowel rest and the institution of total parenteral nutrition (TPN). Bowel rest and TPN are postulated to be beneficial in patients with post-traumatic or postsurgical CA and are effective by fulfilling the nutritional requirement of these patients and decreasing the production of lymph and allowing bowel rest. Total parenteral nutrition is effective in 60 to $80 \%$ cases of CA not responding to dietary modification and should be given for 2 to 6 weeks. Pharmacological management includes diuretics, somatostatin or octreotide and etilefrine. Somatostatin or octreotide has been reported to be useful in some cases and helps in closing fistula more rapidly; they also help in inhibition of lymph fluid excretion through specific receptor found in the normal intestinal wall of the lymphatic vessels. Etilefrine, a sympathomimetic drug that acts by contracting the smooth muscle of the thoracic duct, thereby decreasing the flow of the chyle, is also effective in the management of CA. Embolization of cisterna chyli by fibered endovascular coil, gelatin, sponge, and doxycycline can be tried for management of CA. Surgery is beneficial in patients with postoperative, neoplastic and congenital causes after identifying the site of leakage or the presence of fistula by a lymphangiogram or lymphoscintigraphy. The optimal timing of surgery remains controversial, but most authors recommend a trial of conservative measures for at least 4 to 8 weeks before surgical intervention. Paracentesis should be performed to relieve abdominal discomfort in large and symptomatic ascites and can be repeated as needed, especially in end-stage disease not amenable to medical or surgical management. But paracentesis has been implicated in prolongation of leaks, worsening of protein loss, nutritional depletion and infectious complications. Peritoneovenous shunt like LeVeen or Denver shunt is indicated in patients of refractory CA who are too ill to undergo surgical exploration for direct repair. This is associated with high rate of complications, such as sepsis, disseminated intravascular coagulopathy, electrolyte imbalance, small bowel obstruction and risk for air embolism, and high rate of shunt closure in majority of cases because of the high viscosity of the chyle. ${ }^{1}$

\section{CONCLUSION}

Chylous ascites is a rare and challenging clinical condition that occurs as a result of disruption of abdominal lymphatics. Although malignancy and trauma are most commonly implicated, tuberculosis must always to be considered.

\section{REFERENCES}

1. Frick E, Scholmerich J. Aetiology, diagnosis and management of noncirrhotic ascites. In: Ascites and renal dysfunction in liver diseases. In: Arroyo V, Gines P, Rodes J, Schrier RW, editors. Blackwell Science: Malden; 1999. p. 116-125.

2. Browse NL, Wilson NM, Russo F, Hassan HA, Allen DR. Aetiology and treatment chylous ascites. Br J Surg 1992;79(79): 1145-1150.

3. Press OW, Press NO, Kaufman SD. Evaluation and management of chylous ascites. Ann Intern Med 1982;96(5):358-364.

4. Aalami OO, Allen DB, organ $\mathrm{CH}$ Jr. Chylous ascites: a collective review. Surgery 2000;128(5):761-778.

5. Sathiravikarn W, Apisarnthanarak A, Apisarnthanarak P, Bailey TC. Mycobacterium tuberculosis associated chylous ascites in HIV-infected patients: a case report and review of the literature. Infection 2006;34(4):230-233.

6. Keaveny AP, Karasik MS, Farber HW. Successful treatment of chylous ascites secondary to mycobacterium avium complex in a patient with acquired immune deficiency syndrome. Am J Gastroenterol 1999;94(24):1689-1690.

7. Ekwacani CN. Chylous ascites, tuberculosis and HIV/AIDS: a case report. West Afr J Med 2002;21(24):170-172.

8. Rabie J, Lomp A, Goussard P, Nel E, Cotton M. Paradoxical tuberculosis associated immune reconstruction inflammatory syndrome presenting with chylous ascites and chylothorax in HIV-1 infected child. J Trop Pediatr 2010;56(5):355-358.

9. Cardenas A, Chopra S. Chylous ascites. Am J Gastroenterol 2002;97(8):1896-1900.

10. Vettooretto N, Odeh M, Romessis M, Pettinato G, Taglietti L, Giovanetti M. Acute abdomen from chylous peritonitis: a surgical diagnoisis: case report and literature review. Eur Surg Res 2008;41(29):54-57.

11. Runyon BA. Care of patients with ascites. N Engl J Med 1994;330:3337-3342.

12. Uriz J, Cardenas A, Arroyo V. Pathogenesis diagnosis and treatment of ascites in cirrhosis. Baillieres Best Pract Res Clin Gastroenterol 2000;14:927-943. 